\title{
Development of an Isotope-Dilution Liquid Chromatography/Mass Spectrometric Method for the Accurate Determination of Acetaminophen in Tablets
}

\author{
Hyunju Shin, Byungjoo Kim, Joonhee Lee, and Euijin Hwang \\ Division of Metrology for Quality Life, Korea Research Institute of Standards and Science, Yuseong, \\ Daejeon 305-600, Korea. *E-mail: joonhee@kriss.re.kr \\ Received June 19, 2010, Accepted October 8, 2010
}

\begin{abstract}
Acetaminophen ( $N$-acetyl- $p$-aminophenol) is one of the most popular analgesic and antipyretic drugs. An isotope dilution mass spectrometric method based on LC/MS was developed as a candidate reference method for the accurate determination of acetaminophen in pharmaceutical product. After spiking an isotope labeled acetaminophen (acetyl- ${ }^{13} \mathrm{C}_{2},{ }^{15} \mathrm{~N}-$ acetaminophen) as an internal standard, tablet extracts were analyzed by LC/MS in a selected reaction monitoring (SRM) mode to detect ions at $m / z \quad 152 \rightarrow 110$ and $m / z 155 \rightarrow 111$ for acetaminophen and acetyl $-{ }^{13} \mathrm{C}_{2},{ }^{15} \mathrm{~N}$-acetaminophen, respectively. The repeatability and reproducibility of the developed ID/LC-MS method were tested for the validation and assessment of metrological quality of the method.
\end{abstract}

Key Words: Acetaminophen, ID-LC/MS, Method validation, Pharmaceuticals, Reference method

\section{Introduction}

Acetaminophen (paracetamol, $N$-acetyl- $p$-amino-phenol) is an acylated aromatic amide (Figure 1), which is widely used as analgesic and antipyretic medicine for adults and children. Acetaminophen is one of the non-steroidal anti-inflammatory drugs (NSAIDs), which, in standard doses, are not showing side effects such as stomach irritation or blood coagulation like other common analgesics. Although its safe characteristics, recent studies reported humans and experimental animals exposed to high dose of acetaminophen were associated with hepatic toxicity and renal failure. ${ }^{1,2}$ This shows the importance on tablet composition and uniformity of active compounds in pharmaceutical products.

For the determination of acetaminophen in drugs, many studies reported the applications of various analytical techniques including spectrophotometry, ${ }^{3}$ chemiluminescence, ${ }^{4}$ chromatography, ${ }^{5}$ capillary electrophoresis, ${ }^{6}$ FTIR-spectrometry, ${ }^{7}$ and flow injection analysis (FIA) using UV/VIS spectrophotometer. ${ }^{8}$ Among these techniques, LC with either DAD or MS applications is widely accepted for separating the active compounds in pharmaceutical products $^{9-11}$ or biological samples. ${ }^{2,12}$ An isotope dilution mass spectrometry (IDMS) connecting chromatography is recently believed as one of the high accurate analytical techniques. An ID-LC/MS method is necessary to spike the stable isotope into the sample, to separate the analyte and isotopic compounds from the sample matrix on the LC column and to measure isotope ratio through determination of chromatographic areas for analyte and isotopic compounds. This procedure is able to eliminate the systemic bias through the accurate correction of the recovery of the target analyte along the sample clean-up process. ${ }^{13-15}$ Therefore, IDMS method is identified as one of the potential primary methods in chemistry by CCQM (Comité Consultatif pour la Quantité de Matière). ${ }^{16}$

It is well known that certified reference materials (CRMs) with their certified values traceable to the International System (SI) of Units play a key role for the harmonization of assay

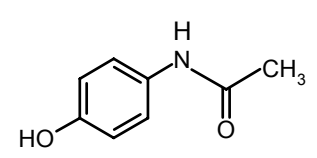

Figure 1. Chemical structure of acetaminophen.

results among laboratories both nationwide and worldwide. The developments of primary analytical methods and reference materials are important role of national measurement institute (NMI) based on concepts of metrological traceability. In order to introduce the reference measurement system in pharmaceutical laboratories, current study established an ID-LC/MS method for the accurate determination of acetaminophen in tablets. The repeatability and reproducibility were tested to validate the developed method and the uncertainty of measurement results was evaluated.

\section{Experimental}

Materials. Acetaminophen (99.6\%) was purchased from U.S. Pharmacopeia (Rockville, MD, USA) as a primary reference standard. Acetyl- ${ }^{13} \mathrm{C}_{2},{ }^{15} \mathrm{~N}$-acetaminophen was purchased from Cambridge Isotope Laboratories, Inc. (Andover, MA, USA). HPLC grade methanol and formic acid were purchased from Burdick and Jackson (Muskegon, MI, USA) and Fluka (St. Gallen, Switzerland), respectively. Filter cartridges (PURDISC NYL 25 FILTER $25 \mathrm{~mm} 0.4 \mu \mathrm{M}$ ) were obtained from Whatman (Clifton, NJ, USA). Analgesic products (tablets) containing acetaminophen were purchased from a local pharmacy.

Calibration standard solutions. Before preparing acetaminophen standard solution, acetaminophen was dried for 18 hours in a desiccator with silica gel as instructed by the manufacturer. To cross-check the standard solution, four independent acetaminophen standard solutions $(100 \mathrm{mg} / \mathrm{kg})$ were gravimetrically prepared in methanol. Isotope labeled acetaminophen, acetyl${ }^{13} \mathrm{C}_{2},{ }^{15} \mathrm{~N}$-acetaminophen, standard solution was also prepared 
in the same way. As a calibration standard solution, isotope ratio standard solution was prepared by mixing acetaminophen standard solution and isotope labeled acetaminophen standard solution (1:1 isotope ratio). Two isotope ratio standard solutions were prepared from each acetaminophen standard solution and all preparation procedures were performed gravimetrically. A portion of the isotope ratio standard was then diluted with methanol to a level which is convenient for LC/ESI/MS analysis $(1 \mathrm{mg} / \mathrm{kg}$ in this study). The eight diluted isotope ratio standards were analyzed by LC/ESI/MS in SIM (selected ion monitoring) mode to cross-check consistencies among them. Repeatability and reproducibility of preparing standard solutions and isotope ratio standards were evaluated by their LC/MS results, and one isotope ratio standard was selected and used for the calibration of sample analysis.

Sample preparation. In order to validate and evaluate metrological quality of the developed method, homogenized sample is required to minimize ambiguity caused by sample in-homogeneity. Homogenized sample was prepared by in-house procedures described as followings. Commercial acetaminophen tablets of a single brand were grinded with a laboratory mill (FRITSCH, Model No. pulverisette 14, Germany). The materials were passed through $100 \mu \mathrm{m}$ nylon sieve cloth and mixed in V-mixer for over 10 hours. Prepared homogenized samples were bottled in $3 \mathrm{~g}$ per unit into $6 \mathrm{~mL}$ amber bottle for further study.

A portion of homogenized sample $(0.2 \mathrm{~g})$ was weighed into a $120 \mathrm{~mL}$ glass bottle and $100 \mathrm{~mL}$ of methanol was added into it. The exact amounts of sample and extraction solvent were determined by weighing the bottle before and after addition of each of them. After sonication for 2 hours in a water bath $(35$ $\left.{ }^{\circ} \mathrm{C}\right), 0.5 \mathrm{~mL}$ of the sample extract was pipetted into a vial for spiking the isotope standard solution. In order to make the isotope ratio as close to $1: 1$, pre-calculated amount of the acetyl${ }^{13} \mathrm{C}_{2},{ }^{15} \mathrm{~N}$-acetaminophen standard solution was spiked to a weighed aliquot of the sample extract. A portion of the isotope spiked sample was passed through a filter cartridge, and then diluted with methanol like as isotope ratio standard $(1 \mathrm{mg} / \mathrm{kg})$.

The developed ID-LC/MS method was applied to four different commercial products containing various levels of acetaminophen as a main constituent. Four tablets from each product were grinded independently and analyzed for acetaminophen as same way of homogenized sample.

Instrumentation and MS analysis. The LC-MS/MS analysis was achieved with Agilent 6410 Triple Quad LC/MS (Santa Clara, CA, USA) connected with Agilent 1200 Series (Waldbronn, Germany) containing a quaternary pump, an autosampler, a degasser, a column oven and a diode-array detector. Data acquisition was performed using an Agilent Mass Hunter Workstation Software (Agilent Corporation, CA, USA).

The chromatographic separation was carried out on a Waters XBridge $\mathrm{C}_{18}(4.6 \mathrm{~mm} \times 150 \mathrm{~mm}, 3.5 \mu \mathrm{m})$ connected with a $\mathrm{C}_{18}$ guard column. Mobile phase was running as isocratic at 0.3 $\mathrm{mL} / \mathrm{min}$ with $100 \%$ of methanol containing $0.1 \%$ formic acid. The injection volume was $10 \mu \mathrm{L}$. MS analysis was conducted in positive mode of electrospray ionization (ESI). In order to obtain the highest precursor ion abundance in the selected ion monitoring (SIM) mode, the following conditions were opti- mized: capillary voltage, $4000 \mathrm{~V}$; nebulizer gas $\left(\mathrm{N}_{2}\right)$ pressure, 45 psi.; drying gas $\left(\mathrm{N}_{2}\right)$ temperature, $350{ }^{\circ} \mathrm{C}$; drying gas flow, 10 $\mathrm{L} / \mathrm{min}$; fragmentor voltage (applied to the extraction skimmer), $100 \mathrm{~V}$. To perform the selected reaction monitoring (SRM) mode, $15 \mathrm{eV}$ of collision energy was applied on the collision cell, and dissociation channels of $m / z 152 \rightarrow 110$ and $\mathrm{m} / z 155 \rightarrow$ 111 were chosen for acetaminophen and isotope labeled acetaminophen, respectively. SIM mode monitored $[\mathrm{M}+\mathrm{H}]^{+}$ions of acetaminophen and acetyl- ${ }^{13} \mathrm{C}_{2},{ }^{15} \mathrm{~N}$-acetaminophen at $\mathrm{m} / \mathrm{z} 152$ and 155 , respectively.

Calculation. The mass fractions of acetaminophen in test samples $(\%$ in $\mathrm{kg} / \mathrm{kg})$ were determined by the isotope ratio of acetaminophen/acetyl- ${ }^{13} \mathrm{C}_{2},{ }^{15} \mathrm{~N}$-acetaminophen in isotope spiked sample comparing isotope ratio standard. ${ }^{13-15,20}$

$$
\boldsymbol{C}=\frac{\boldsymbol{M}_{\text {is-sol, spiked }} \times \boldsymbol{A} \boldsymbol{R}_{\text {sample }} \times \boldsymbol{M}_{\text {s-sol, std }} \times \boldsymbol{C}_{s-\text { sol }}}{\boldsymbol{W}_{s} \times \boldsymbol{A} \boldsymbol{R}_{\text {std }} \times \boldsymbol{M}_{\text {is-sol, std }}}
$$

where:

$\boldsymbol{M}_{i s-\text { sol, spiked }}$ is the mass of the acetyl- ${ }^{13} \mathrm{C}_{2},{ }^{15} \mathrm{~N}$-acetaminophen solution spiked into tablet sample solution; $\boldsymbol{A R}_{\text {sample }}=\boldsymbol{I}_{\mathrm{s} \text {,sample }} /$ $\boldsymbol{I}_{\text {is,spiked }}$ is the observed area ratio of acetaminophen/acetyl${ }^{13} \mathrm{C}_{2},{ }^{15} \mathrm{~N}$-acetaminophen for the sample solution from LC/MS measurement; $\boldsymbol{M}_{s-\text { sol, std }}$ is the mass of the acetaminophen solution spiked into isotope ratio standard solution; $\boldsymbol{C}_{s-\text { sol }}$ is the concentration of the acetaminophen solution; $\boldsymbol{W}_{s}$ is the mass of the sample taken for analysis; $\boldsymbol{A} \boldsymbol{R}_{s t d}=\boldsymbol{I}_{s, s t d} / \boldsymbol{I}_{i s, s t d}$ is the observed area ratio of acetaminophen/acetyl- ${ }^{13} \mathrm{C}_{2},{ }^{15} \mathrm{~N}$-acetaminophen for isotope ratio standard solution from LC/MS measurement; $\boldsymbol{M}_{\text {is-sol,std }}$ is the mass of the acetyl- ${ }^{13} \mathrm{C}_{2},{ }^{15} \mathrm{~N}$-acetaminophen solution spiked into isotope ratio standard solution.

\section{Results and Discussion}

LC/MS optimization. For better LC performance, mobile phase was finalized to $100 \%$ methanol containing $0.1 \%$ formic acid and column was chosen for Waters XBridge $\mathrm{C}_{18}$ column. Prior to determine the methanol as a mobile phase, various mobile phases were tested to obtain better chromatographic separation and higher sensitivity by LC/MS analysis. Methanol was an organic solvent, and ammonium acetate buffer $(10 \mathrm{mmol} / \mathrm{L})$ and ammonium formate buffer $(10 \mathrm{mmol} / \mathrm{L})$ were tested as an aqueous solvent. Acetaminophen showed proper retention and peak shape when the mobile phase contained $40 \%$ of either of the two buffer solutions. However, when the aqueous phase composition was above $40 \%$ of the mobile phase, LC/MS performance for acetaminophen showed irregular retention and peak broadening with low ionization efficiency. The $\mathrm{pH}$ of mobile phase did not influence acetaminophen behaviors on the column which was accorded with previous observations that increasing $\mathrm{pH}$ did not affect neutral compounds, such as acetaminophen or chloracetanilide. ${ }^{17}$ Figure $2 \mathrm{~A}-\mathrm{C}$ presents LC/MS chromatograms of acetaminophen with various aqueous solvents showing that the ionization efficiency with $40 \%$ of either ammonium acetate buffer or ammonium formate buffer was lower comparing those by $40 \%$ water. However, the best peak shape and LC/MS intensity were produced with $100 \%$ methanol containing $0.1 \%$ formic 


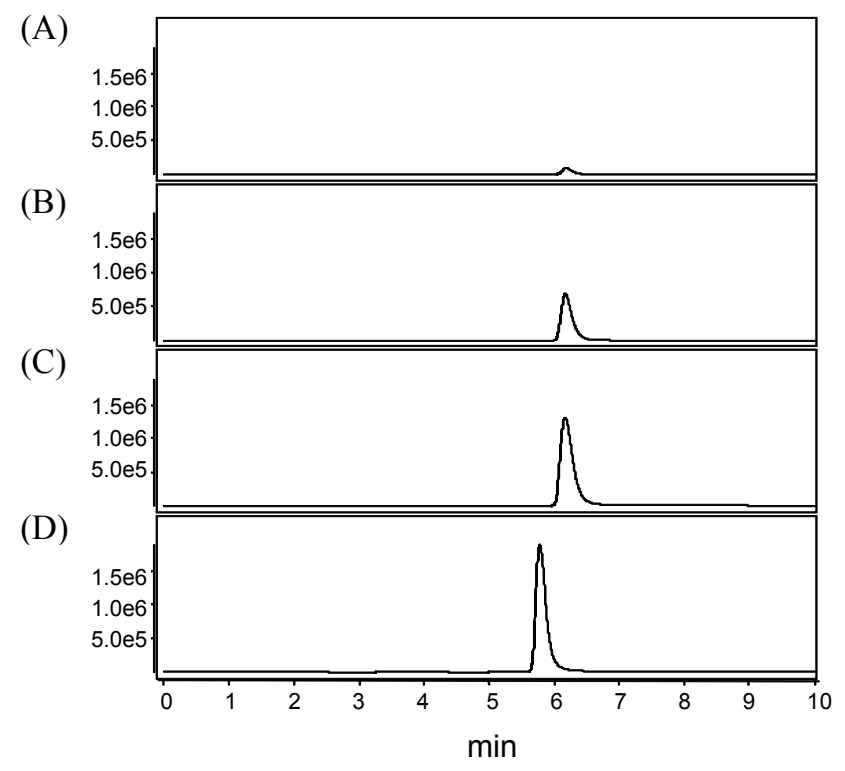

Figure 2. Total ion chromatograms produced with various isocratic mobile phases compositions (A: $10 \mathrm{mmol} / \mathrm{L}$ ammonium acetate $(\mathrm{pH} 7)-$ $\mathrm{MeOH}(40: 60, \mathrm{v} / \mathrm{v}), \mathrm{B}: 10 \mathrm{mmol} / \mathrm{L}$ ammonium formate $(\mathrm{pH} 7)-\mathrm{MeOH}$ $(40: 60, \mathrm{v} / \mathrm{v}), \mathrm{C}$ : water-MeOH (40:60, v/v), D: $\mathrm{MeOH}$ containing $0.1 \%$ formic acid).

acid (Figure 2 D), and this solvent at $0.3 \mathrm{~mL} / \mathrm{min}$ was finally chosen as a mobile phase for sample analysis.

Mass spectrometer was operated in positive mode and fullscan mass spectra of acetaminophen and acetyl ${ }^{13} \mathrm{C}_{2},{ }^{15} \mathrm{~N}$-acetaminophen were dominated by $[\mathrm{M}+\mathrm{H}]^{+}$at $m / z 152$ and 155 , respectively. Collisionally induced dissociation (CID) produced $\left[\mathrm{M}+\mathrm{H}-\mathrm{COCH}_{3}\right]^{+}$as a dominant product ion (Figure 3), which accords with published data. ${ }^{2}$ SRM mode was applied to determine analytes by monitoring the transition of $\mathrm{m} / \mathrm{z} 152 \rightarrow 110$ and $\mathrm{m} / \mathrm{z} 155 \rightarrow 111$ for acetaminophen and acetyl $-{ }^{13} \mathrm{C}_{2},{ }^{15} \mathrm{~N}$-acetaminophen, respectively. Figure 4 shows LC SRM chromatograms of acetaminophen and acetyl- ${ }^{13} \mathrm{C}_{2},{ }^{15} \mathrm{~N}$-acetaminophen from a pharmaceutical tablet. Comparing SIM and SRM modes, both detection modes provide similar analytical quality for determination of acetaminophen by ID-LC/MS. In this study, SRM mode was chosen for the quantitative analysis of acetaminophen by ID-LC/MS as it has one more stage of selectivity compared to SIM mode. However, we also tested SIM mode in parallel with SRM mode along this study.

Repeatability and reproducibility. It is essential to confirm that the developed ID-LC/MS method produce reliable measurement results for the evaluation of acetaminophen contents in pharmaceutical materials. Due to the limit of available wellcharacterized CRMs related with acetaminophen, homogenized samples were prepared in the way of in-house reference material production as described in the section of sample preparation. Figure 5 shows the mean results for each 10 sub-samples of produced homogenized sample. The error bars represent the expanded uncertainty of each value [see next session for uncertainty evaluation]. An average acetaminophen contents was $40.6 \%$ in $\mathrm{kg} / \mathrm{kg}$ and a relative standard deviation among the ID-LC/MS measurement results on the 10 subsamples of homogenized sample was $1.0 \%$ of the average, which represents sum of the
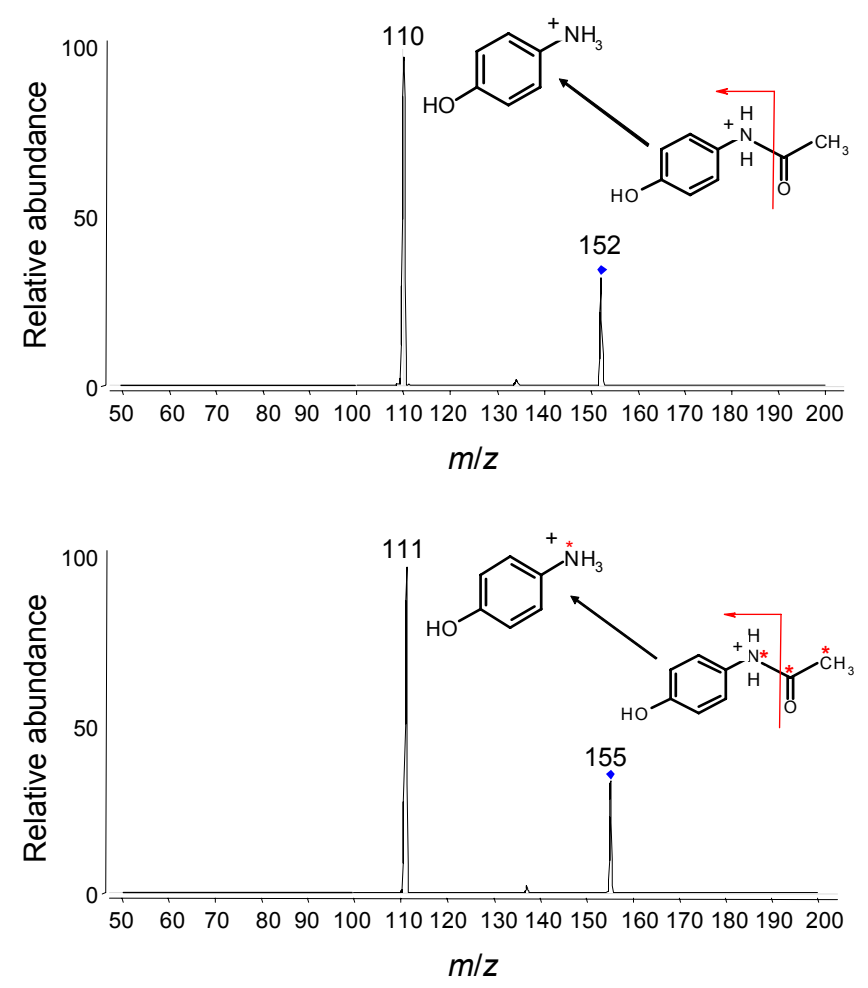

Figure 3. Product ion spectra of collisionally induced dissociation (CID) of the $[\mathrm{M}+\mathrm{H}]^{+}$ions of acetaminophen and acetyl- ${ }^{13} \mathrm{C}_{2},{ }^{15} \mathrm{~N}$-acetaminophen. Dissociation mechanism of the dominant channel, $[\mathrm{M}+\mathrm{H}]^{+}$ ions to $\left[\mathrm{M}+\mathrm{H}-\mathrm{COCH}_{3}\right]^{+}$, was schematically shown on the mass spectra.

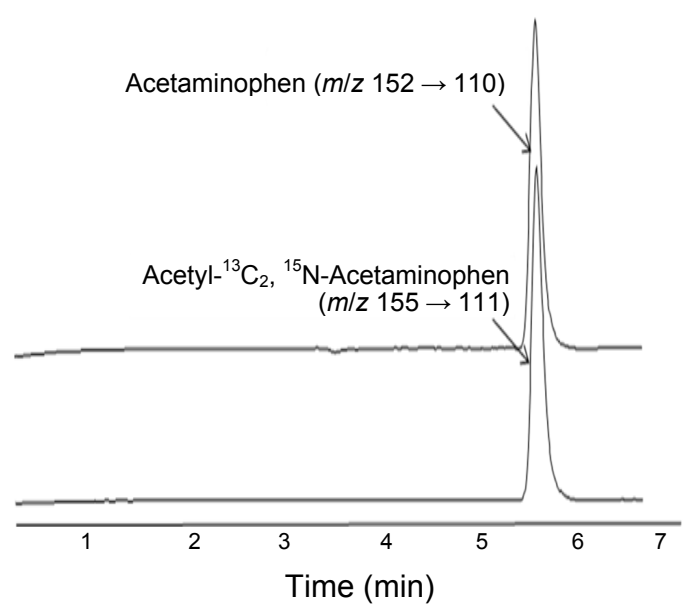

Figure 4. Typical SRM chromatograms of acetaminophen $(\mathrm{m} / \mathrm{z} 152 \rightarrow$ $110)$ and acetyl $-{ }^{13} \mathrm{C}_{2},{ }^{15} \mathrm{~N}$-acetaminophen $(\mathrm{m} / z 155 \rightarrow 111)$ in isotope spiked sample extracts.

repeatability of the measurement method and possible sample in-homogeneity. Table 1 summarized the measurement results obtained at three different time periods. In order to test the reproducibility, a new set of multiple standard solutions were prepared and verified by the consistency test among them as described in experimental section. The mean averages of measurement results in each time period were $40.6,41.0$, and $40.4 \%$ in $\mathrm{kg} / \mathrm{kg}$, respectively, and the relative standard deviation within 


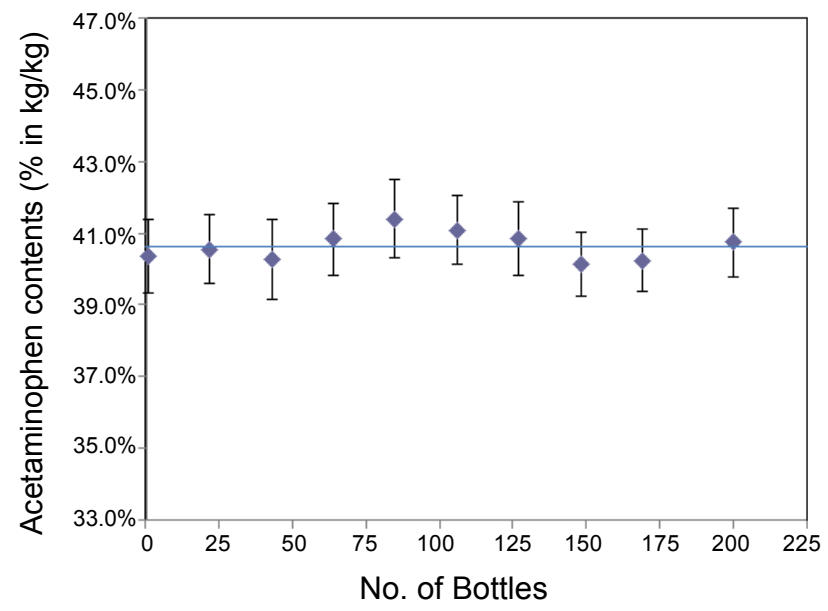

Figure 5. Graphical view of acetaminophen contents (\% in $\mathrm{kg} / \mathrm{kg})$ in 10 bottles of homogenized sample (Error bars represents the relative expanded uncertainty of measurement results).

a period was between 0.3 and $0.4 \%$, indicating that the good repeatability was maintained for long-term period. The developed ID-LC/MS improved the repeatability comparing other analytical techniques such as LC-UV ${ }^{10,11}$ or $\mathrm{CE},{ }^{18}$ which showed the ranges of $0.76 \sim 2.26 \%$ for repeatability. Measurement results from the three different time periods show around $0.8 \%$ of relative standard deviation and agree to each other within their uncertainties, indicating that the method has a good reproducibility in tested time periods.

Application on commercial tablets. Commercial analgesic products, containing different levels of acetaminophen as a main active ingredient, were evaluated by the developed ID-LC/MS method in both SRM and SIM modes (Table 2). The results from the SRM and the SIM modes agreed within their uncertainties. According to our observation, the relative standard deviation of measurement results among tablets of each single products was less than $0.6 \%$, and it shows that the difference in acetaminophen contents between tablets are not significant for the products tested in this study.

Uncertainty sources. The uncertainty estimation is internationally accepted as a means to confirm the reliability of measurement results as described in the Guide to the Expression of Uncertainty in Measurement (GUM). ${ }^{19}$ The ISO Guide recommend to express uncertainty as an expanded uncertainty, $U$, which is associated with all the expected uncertainty components during the whole process of measurement. Finally, $U$ is calculated by combined uncertainty sources $\left(u_{c}\right)$ multiple with coverage factor, $k$, which is determined from the Student's $t$-distribution corresponding to the appropriate associated degrees of freedom and an approximate $95 \%$ confidence for each analyte, namely $U=k u_{c}$. The uncertainty evaluation in this study was referred to previously published papers ${ }^{20,21}$ and GUM. ${ }^{19}$ The uncertainty components in ID-LC/MS measurement were categorized into systematic and random effects according to their effects on the variation of multiple measurements. ${ }^{20,21}$ Details on the evaluation of uncertainty of results from multiple measurement by IDMS method was described in another article, ${ }^{21}$ and a brief description is given here. Table 3 lists sources of uncer-
Table 1. Measurement results of acetaminophen in a homogenized sample by ID-LC/MS in the SRM mode during three different time periods

\begin{tabular}{|c|c|c|}
\hline & Subsample No. & $\begin{array}{l}\text { Measurement Results } \\
(\% \text { in } \mathrm{kg} / \mathrm{kg})\end{array}$ \\
\hline \multirow{7}{*}{ Period 1} & $\# 1$ & $40.7^{a}$ \\
\hline & $\# 2$ & 40.4 \\
\hline & $\# 3$ & 40.7 \\
\hline & $\# 4$ & 40.6 \\
\hline & Average & 40.6 \\
\hline & Standard deviation & $0.1\left(0.3 \mathrm{rel} \%{ }^{b}\right)$ \\
\hline & Expanded uncertainty & $0.4\left(1.0 \mathrm{rel} \%^{b}\right)$ \\
\hline \multirow{7}{*}{ Period 2} & $\# 1$ & 41.1 \\
\hline & $\# 2$ & 40.9 \\
\hline & $\# 3$ & 41.0 \\
\hline & $\# 4$ & 41.2 \\
\hline & Average & 41.0 \\
\hline & Standard deviation & $0.1\left(0.3 \mathrm{rel} \%{ }^{b}\right)$ \\
\hline & Expanded uncertainty & $0.3\left(0.7 \mathrm{rel} \%^{b}\right)$ \\
\hline \multirow{7}{*}{ Period 3} & $\# 1$ & 40.2 \\
\hline & $\# 2$ & 40.4 \\
\hline & $\# 3$ & 40.3 \\
\hline & $\# 4$ & 40.6 \\
\hline & Average & 40.4 \\
\hline & Standard deviation & $0.2\left(0.4 \mathrm{rel} \%{ }^{b}\right)$ \\
\hline & Expanded uncertainty & $0.4\left(1.0 \mathrm{rel} \%^{b}\right)$ \\
\hline \multirow{2}{*}{\multicolumn{2}{|c|}{$\begin{array}{c}\text { Average } \\
\text { Standard deviation among period }\end{array}$}} & 40.7 \\
\hline & & $0.3\left(0.8\right.$ rel $\left.\%^{b}\right)$ \\
\hline
\end{tabular}

${ }^{a}$ Each measurement result was expressed as mean of 4 injections. ${ }^{b}$ The unit "rel \%" indicates the relative percentage of the standard deviation or the uncertainty of the value in front of the parenthesis in comparison with the corresponding mean value.

Table 2. Measurement results of acetaminophen in commercial pharmaceutical products by ID-LC/MS running at SRM and SIM modes

\begin{tabular}{ccc}
\hline \multirow{2}{*}{ Products } & \multicolumn{2}{c}{ Acetaminophen Contents $(\%$ in $\mathrm{kg} / \mathrm{kg})$} \\
\cline { 2 - 3 } & SRM mode & SIM mode \\
\hline Product A & $50.9 \pm 2.2^{a}$ & $51.6 \pm 2.7$ \\
Product B & $71.5 \pm 0.6$ & $71.3 \pm 0.6$ \\
Product C & $77.3 \pm 0.7$ & $77.2 \pm 1.0$ \\
Product D & $78.6 \pm 1.0$ & $78.4 \pm 0.7$ \\
\hline
\end{tabular}

${ }^{a}$ The values following " \pm " are the expanded uncertainties of the preceding values at the $95 \%$ level of confidence. For each product, four tablets were analyzed separately, and the uncertainty includes the standards deviation of measurement results among tablets.

tainty in measurements of acetaminophen in pharmaceutical tablets using by ID-LC/MS. Group I $\left(u_{s y s}\right)$ includes the uncertainty components which systematically affect to the measurement results, for example the uncertainties of standard solution, isotope ratio standard, and the peak area ratio of acetaminophen/ acetyl $-{ }^{13} \mathrm{C}_{2},{ }^{15} \mathrm{~N}$-acetaminophen from LC/MS measurements of 
Table 3. Uncertainty sources in the ID-LC/MS determination of acetaminophen in pharmaceutical products

\begin{tabular}{|c|c|c|c|}
\hline Group & Uncertainty Components & $\begin{array}{c}\text { Sources } \\
\text { (Evaluation Methods) }\end{array}$ & $\begin{array}{c}\text { Typical } \\
\text { value }^{a} \\
\text { (Relative \%) }\end{array}$ \\
\hline \multirow{4}{*}{$I^{b}$} & \multirow[b]{2}{*}{ Standard solution } & Purity of the reference material (from the certificate) & 0.3 \\
\hline & & $\begin{array}{l}\text { Gravimetric preparation (from cross-check of indepen- } \\
\text { dent sets of calibration solutions) }\end{array}$ & 0.1 \\
\hline & Isotope ratio standard & $\begin{array}{l}\text { Gravimetric mixing (from cross-check of multiple iso- } \\
\text { tope ratio standards from each individual standard } \\
\text { solution) }\end{array}$ & 0.2 \\
\hline & $\begin{array}{l}\text { Peak area ratio of acetaminophen and acetyl }-{ }^{13} \mathrm{C}_{2},{ }^{15} \mathrm{~N} \text {-acetamino- } \\
\text { phen from LC/MS measurements of isotope ratio standard }\end{array}$ & Standard deviation of multiple measurements & 0.2 \\
\hline \multirow{3}{*}{$\mathrm{II}^{c}$} & Mass of sample taken for analysis & $\begin{array}{l}\text { Readability and linearity of the balance used (from } \\
\text { the certificate of the balance) }\end{array}$ & $<0.01$ \\
\hline & $\begin{array}{l}\text { Mass of acetyl- }{ }^{13} \mathrm{C}_{2},{ }^{15} \mathrm{~N} \text {-acetaminophen solution spiked into } \\
\text { sample taken for analysis }\end{array}$ & $\begin{array}{l}\text { Readability and linearity of the balance used (from } \\
\text { the certificate of the balance) }\end{array}$ & $<0.01$ \\
\hline & $\begin{array}{l}\text { Peak area ratio of acetaminophen and acetyl }{ }^{13} \mathrm{C}_{2},{ }^{15} \mathrm{~N} \text {-acetamino- } \\
\text { phen from LC/MS measurements of sample extract }\end{array}$ & Standard deviation of multiple measurements & $0.1-0.3$ \\
\hline
\end{tabular}

${ }^{a}$ Typical uncertainty of each source is based on the measurement protocol used in this study. ${ }^{b}$ Group I includes the uncertainty components common to the measurement of all bottles (Systematic effect, $u_{s y s}$ ). ${ }^{c}$ Group II includes the uncertainty components that are unique to each bottle (Random effect, $u_{r a n}$ ).

isotope ratio standard. Group II $\left(u_{r a n}\right)$ is associated with uncertainty components of weighing sample taken for analysis, weighing acetyl- ${ }^{13} \mathrm{C}_{2},{ }^{15} \mathrm{~N}$-acetaminophen solution spiked to the sample, and peak area ratios of isotope ratio standard and sample extracts from LC/MS measurements. The overall uncertainty is evaluated by combining all the uncertainty components in Group I and II. Therefore, the relative standard uncertainty of the measurement value for acetaminophen in tablets was expected to be $1.0 \%$, calculated following equation, ${ }^{19-21} u(C$ mean $)=$ $\sqrt{u^{2}{ }_{\text {sys }}+u_{\text {ran }}^{2}}$. This level of low uncertainty supports that the method has a high metrological quality as a reference method.

\section{Conclusions}

An isotope dilution mass spectrometric method has been explored and established to measure the acetaminophen contents ( $\%$ in $\mathrm{kg} / \mathrm{kg})$ in pharmaceutical products. The repeatability/reproducibility studies and the uncertainty evaluation results have proven that the candidate method has a metrological quality which is adequate enough to be used as a reference method.

\section{References}

1. Bosch, M. E.; Sánchez, A. J. R.; Rojas, F. S.; Ojeda, C. B. J. Pharm. Biomed. Anal. 2006, 42, 291.

2. Chen, X.; Huang, J.; Kong, Z.; Zhong, D. J. Chromtogr. B 2005, $817,263-269$.
3. Hanaee, J. Pharm. Acta Helv. 1997, 72, 239.

4. Zhao, S.; Bai, W.; Yuan, H.; Xiao, D. Anal. Chim. Acta 2006, 559, 195.

5. Ravisankar, S.; Vasudevan, M.; Gandhimathi, M.; Suresh, B. Talanta 1998, 46, 1577.

6. He, F. Y.; Liu, A. L.; Xia, X. H. Anal. Bioanal. Chem. 2004, 379, 1062.

7. Ramos, M. L.; Tyson, J. F.; Curran, D. J. Anal. Chim. Acta 1998, $364,107$.

8. Bouhsain, Z.; Garrigues, S.; Morales-Rubio, A.; de la Guardia, M. Anal. Chim. Acta 1996, 330, 59.

9. Marín, A.; Barbas, C. J. Pharm. Biomed. Anal. 2004, 35, 1035.

10. García, A.; Rupérez, F. J.; Marín, A.; de la Maza, A.; Barbas, C. J. Chromtogr. B 2005, 785, 237.

11. Marín, A.; García, E.; García, A.; Barbas, C. J. Pharm. Biomed. Anal. 2002, 29, 701.

12. Zhang, Y.; Mehrotra, N.; Budha, N. R.; Christense, M. L.; Meibhm, B. Clinica Chimica Acta 2008, 398, 105.

13. Dube, G.; Henrion, A.; Richter, W. Metrologia 1997, 34, 83.

14. De Bivère, P. Anal. Proc. 1993, 30, 328.

15. Jung, P. G.; Kim, B.; Park, S.-R.; So, H.-Y.; Shi, L. H.; Kim, Y. Anal. Bioanal. Chem. 2004, 380, 782-788.

16. Quinn, T. J. Metrologia 1997, 34, 61.

17. Marín, A.; Barbas, C. J. Pharm. Biomed. Anal. 2006, 40, 262.

18. Marín, A.; Barbas, C. J. Pharm. Biomed. Anal. 2004, 35, 769.

19. BIPM, IEC, IFCC, ISO, IUPAC, IUPAP and OIML Guide to the Expression of Uncertainty in Measurement (Geneva, Switzerland: International Organization for Standardization) ISBN 92-6710188-9 1995.

20. Kim, B.; Park, S.; Lee, I.; Lim, Y.; Hwang, E.; So, H.-Y. Anal. Bioanal. Chem. 2010, 398, 1035.

21. Kim, B.; Hwang, E.; So, H.-Y.; Son, E. K. Bull. Korean Chem. Soc. 2010, 31, 3139 . 